\title{
Direct admission versus secondary transfer for mechanical thrombectomy: long-term clinical outcomes from a single Polish Comprehensive Stroke Centre
}

\author{
Piotr Luchowski®i ${ }^{1}$, Joanna Wojczal ${ }^{1}$, Katarzyna Prus ${ }^{1}$, Maciej Szmygin ${ }^{2}$, Michał Sojka ${ }^{2}$, \\ Elżbieta Luchowska $\mathbb{1}^{3}$, Konrad Rejdak $\mathbb{1}^{1}$ \\ ${ }^{1}$ Department of Neurology, Medical University of Lublin, Poland \\ ${ }^{2}$ Department of Interventional Radiology and Neuroradiology, Medical University of Lublin, Poland \\ ${ }^{3}$ Department of Laboratory Diagnostics Medical University of Lublin, Poland
}

\begin{abstract}
Introduction. We aimed to compare 3-month clinical outcomes after mechanical thrombectomy (MT) in patients transferred directly to a comprehensive stroke centre ('mothership', MS) to the outcomes of patients transferred secondarily from primary stroke centres ('drip-and-ship', DAS) in Lubelskie province, the third largest province in Poland.

Material and methods. In a prospective stroke registry, all patients with large vessel occlusion in anterior circulation admitted within six hours of onset and treated with MT between 2017 and 2020 were retrospectively analysed.

Results. A total of 400 patients was evaluated: 267 treated with the MS approach and 133 with the DAS approach. Time from stroke onset to groin puncture was shorter in the MS group. There was a significant difference in 3-month excellent clinical outcomes (mRS $0-1$ ) between these two groups (32.9\% of MS patients vs. $22.5 \%$ of DAS patients, $p<0.05$ ), but there was no difference if the 3-month endpoint was expressed as mRS $\leq 2(42.3 \%$ of MS vs. $34.5 \%$ of DAS patients, $p=0.13)$. The rate of symptomatic intracranial haemorrhage and mortality was comparable in both groups.

Conclusions. Our study shows that direct admission to a comprehensive stroke centre resulted in more patients achieving excellent treatment outcomes (mRS 0-1). At the same time, the superiority of the MT model over the DAS model in obtaining mRS $0-2$ was not unequivocally demonstrated. Further studies are needed to determine the best stroke model for patients potentially eligible for MT.

Key words: mothership, drip and ship, acute ischaemic stroke, mechanical thrombectomy, regional stroke care
\end{abstract}

(Neurol Neurochir Pol 2021; 55 (5): 494-498)

\section{Introduction}

Different prehospital referral systems have been proposed for patients with acute ischaemic stroke (AIS) who are potential candidates for mechanical thrombectomy (MT). The two most widely known are the drip-and-ship (DAS) and the mothership (MS) models [1].

The DAS model consists of transferring the patient to the nearest primary stroke centre (PSC), where thrombolysis therapy (IVT) is initiated, followed by a transfer of patients who are candidates for MT to a comprehensive stroke centre (CSC). The
MS paradigm is direct transfer to a CSC, bypassing the nearest PSC. Both systems have been evaluated in a few observational studies, but as yet the results of only one randomised trial are available [2]. Recently published meta-analysis showed that patients with AIS eligible for reperfusion strategies might benefit more from the MS model than from the DAS model [3]. Furthermore, one recent study has demonstrated lower disability and mortality among patients with large vessel occlusion [LVO] who were directly transported to the CSC if the additional delay was $<30$ minutes and $<50$ minutes in urban and rural areas, respectively [4].

Address for correspondence: Piotr Luchowski, Medical University of Lublin, Kazimierza Jaczewskiego Str. 8, 20-954 Lublin, Poland; e-mail: piotr.luchowski@umlub.pl Received: 5.04.2021 Accepted: 2.07.2021 Early publication date: 23.07.2021

This article is available in open access under Creative Common Attribution-Non-Commercial-No Derivatives 4.0 International (CC BY-NC-ND 4.0) license, allowing to download articles and share them with others as long as they credit the authors and the publisher, but without permission to change them in any way or use them commercially. 
On the other hand, the only completed randomised controlled trial (the RACECAT trial) does not support the hypothesis that direct triage of patients with symptoms of ischaemic stroke due to LVO to CSC leads to improved outcomes compared to the current practice of transferring all patients to the PSC [2].

This explains why the debate as to the best stroke strategy is ongoing, and why there are no clear recommendations in the current stroke guidelines.

The CSC located in the Public Clinic Hospital no. 4 (SPSK4) in Lublin, Poland forms a stroke network with 12 PSCs where only IVT is available. This study aimed to compare the onsetto-groin time, reperfusion rate, symptomatic intracranial haemorrhage (sICH) and mortality rate, as well as clinical outcome at 3 months after MT, in direct CSC admission AIS patients $v s$. secondarily transferred AIS patients.

\section{Material and methods}

In this single-centre study, we performed a retrospective analysis of prospectively collected data of 400 consecutive patients admitted with acute ischaemic stroke due to LVO of anterior circulation who underwent endovascular thrombectomy between January 2017 and November 2020. The first group included patients directly arriving at CSC at SPSK4 in Lublin (we called this the mothership model, MS), and the second group consisted of patients admitted to one of the above-mentioned hospitals with secondary transfer to the CSC (we called this the drip-and-ship model, DAS). Average distance from PSCs to CSC is $50 \mathrm{~km}$.

The following inclusion criteria were applied: 1) LVO of anterior circulation confirmed by imaging examination (non-contrast CT and CT-angio and/or MRI) and treated with MT; 2) time from symptoms onset to reperfusion of no more than $6 \mathrm{~h}$; 3) National Institute of Health Stroke Scale (NIHSS) score $\geq 6 ; 4)$ no prestroke dependency expressed as modified Rankin Scale (mRS) score $0-2$.

Clinical data including age, sex, stroke risk factors, baseline medication, initial laboratory results, stroke severity as expressed by the NIHSS score, and time metrics were collected and evaluated. In accordance with the current Guidelines of the Polish Neurological Society for the Management of Patients with Ischaemic Stroke, intravenous thrombolysis (rt-PA) was administered if patients arrived in a window time $<4.5 \mathrm{~h}$ and where there was no contraindication [5]. An institutional review committee approved this study (approval number KE-0254/285/2019.). This study was conducted in accordance with the Declaration of Helsinki.

\section{Endovascular thrombectomy}

All procedures were performed under biplane angiography unit with 3D rotational angiography and with patients under conscious sedation or general anaesthesia. Mechanical thrombectomy was carried out with aspiration (ACE, Penumbra, Alameda), stent retriever (Solitaire, EV3, Irvine, CA, USA) or a combination of both (the Solumbra technique). Final recanalisation was assessed according to the Thrombolysis in Cerebral Infarction (TICI) classification. Good recanalisation was defined as TICI $2 \mathrm{~b}$ and TICI 3 . Complications related to the procedure were noted.

\section{Follow-up}

Routine non-contrast brain CT was performed $24 \mathrm{~h}$ after the procedure to evaluate brain infarction and assess the occurrence of intracranial haemorrhage (ICH) if available. ICH was classified as symptomatic according to the classification of the European-Australasian Acute Stroke Study (ECASS II) [6]. Clinical outcome was assessed based on the mRS score 90 days after the procedure. An excellent and a favourable result were defined as $m R S 0-1$ and $m R S \leq 2$, respectively. Mortality rate was calculated.

\section{Statistical analysis}

Statistical analysis was conducted using a StatSoft Statistica 13.1PL package. The patients were classified into two groups (MS patients $v s$. DAS patients) and comparisons were made in terms of demographic data, initial NIHSS, risk factors, use of IVT, procedural details, and outcomes. Student's t-test, Mann-Whitney test and Chi-squared Pearson tests were used when appropriate. Statistical significance was defined as $\mathrm{p} \leq 0.05$.

\section{Results}

During the study period (January 2017-November 2020), 400 AIS patients (267 MS patients and 133 DAS patients) with LVO in the anterior circulation were treated with MT. There was no significant difference between the groups with regards to baseline clinical characteristics or initial neurological deficit. Furthermore, IVT rate was comparable in both groups ( $74.5 \%$ in MS vs. $81.9 \%$ in DAS; $\mathrm{p}=0.09$ ). Time from picture-to-puncture and onset-to-groin puncture was significantly shorter in the MS group $(61.5 \pm 26.5 \mathrm{~min}$ for MS and $145.8 \pm 67.0 \mathrm{~min}$ for DAS patients, $\mathrm{p}<0.001$ and 178.7 $\pm 64.5 \mathrm{~min}$ in MS patients $v s .263 .7 \pm 58.4 \mathrm{~min}$ in DAS patients, $\mathrm{p}<0.001$, respectively), whereas door-to-groin puncture time was shorter in the DAS group (median $35.4 \pm 25.2 \mathrm{~min} v s$. $85.9 \pm 44.2 \mathrm{~min}, \mathrm{p}<0.001)$.

The rate of successful recanalisation (TICI $2 b-3$ ) as well as failed recanalisation (TICI 0 ) at the end of the procedure was similar in both groups (69.6\% for MS patients vs. $68.4 \%$ for DAS patients; $\mathrm{p}=0.799$ for TICI $2 \mathrm{~b}-3$ and $10.4 \%$ for MS patients $v$ s. $9.8 \%$ for DAS patients; $\mathrm{p}=0.825$ for TICI 0 ). The clinical and procedural details are set out in Table 1.

There was a significant difference in the 3-month excellent clinical outcomes expressed as mRS $0-1$ between these two groups (32.9\% of MS patients $v$ s. $22.5 \%$ of DAS patients, $\mathrm{p}<0.05)$. Similarly, a greater number of patients achieved mRS 0-2 in the MS model compared to the DAS model, although statistical significance was not reached (42.3\% MS vs. $34.5 \%$ 
Table 1. Baseline and treatment characteristics. Statistical significance marked in bold

\begin{tabular}{|c|c|c|c|}
\hline Characteristic & MS group $(n=267)$ & DAS group ( $n=133$ ) & P-value \\
\hline \multicolumn{4}{|l|}{ Baseline } \\
\hline Males (n, \%) & $114(42.7 \%)$ & $66(49.6 \%)$ & 0.18 \\
\hline Age, y (mean) & $73.7 \pm 11.9$ & $72.3 \pm 12.5$ & 0.16 \\
\hline mRS score $=0-1$ & $211(79.0 \%)$ & $113(85.0 \%)$ & 0.15 \\
\hline $\mathrm{mRS}$ score $=2$ & $56(21.0 \%)$ & $20(15.0 \%)$ & 015 \\
\hline Hypertension (n, \%) & $137(51.3 \%)$ & $59(44.4 \%)$ & 0.19 \\
\hline Diabetes (n, \%) & $75(28.1)$ & $41(30.8 \%)$ & 0.57 \\
\hline Atrial fibrillation (n, \%) & $56(21.0)$ & $30(22.6)$ & 0.72 \\
\hline Baseline NIHSS & $18.2 \pm 5.4$ & $20.1 \pm 4.1$ & 0.11 \\
\hline IV-thrombolysis (n, \%) & $199(74.5 \%)$ & $109(81.9 \%)$ & 0.09 \\
\hline \multicolumn{4}{|l|}{ Time in min (mean \pm SD) } \\
\hline Picture-to-puncture & $61.5 \pm 26.5$ & $145.8 \pm 67.0$ & $<0.001$ \\
\hline Onset-to-puncture & $178.7 \pm 64.5$ & $263.7 \pm 58.4$ & $<0.001$ \\
\hline Door-to-puncture & $85.9 \pm 44.2$ & $35.4 \pm 25.2$ & $<0.001$ \\
\hline \multicolumn{4}{|l|}{ Procedural results (n, \%) } \\
\hline Recanalisation ( $\mathrm{TICl} 2 \mathrm{~b}-3$ ) & $186(69.6 \%)$ & $91(68.4 \%)$ & 0.79 \\
\hline No recanalisation $(\mathrm{TICl} 0)$ & $28(10.4 \%)$ & $13(9.8 \%)$ & 0.83 \\
\hline sICH & $23(8.5 \%)$ & $11(7.9 \%)$ & 0.10 \\
\hline \multicolumn{4}{|l|}{ Clinical outcome (n, \%) } \\
\hline NIHSS at discharge & 7.51 & 8.47 & 0.21 \\
\hline 3-months mRS score $=0-1$ & $88(32.9 \%)$ & $30(22.5 \%)$ & $<0.05$ \\
\hline 3-months mRS score $\leq 2$ & $113(42.3 \%)$ & $46(34.5 \%)$ & 0.13 \\
\hline 3-months mortality rate & $57(21.4 \%)$ & $31(23.1 \%)$ & 0.44 \\
\hline
\end{tabular}

Used tests: Student's t-test, Mann-Whitney test, Chi-squared Pearson test

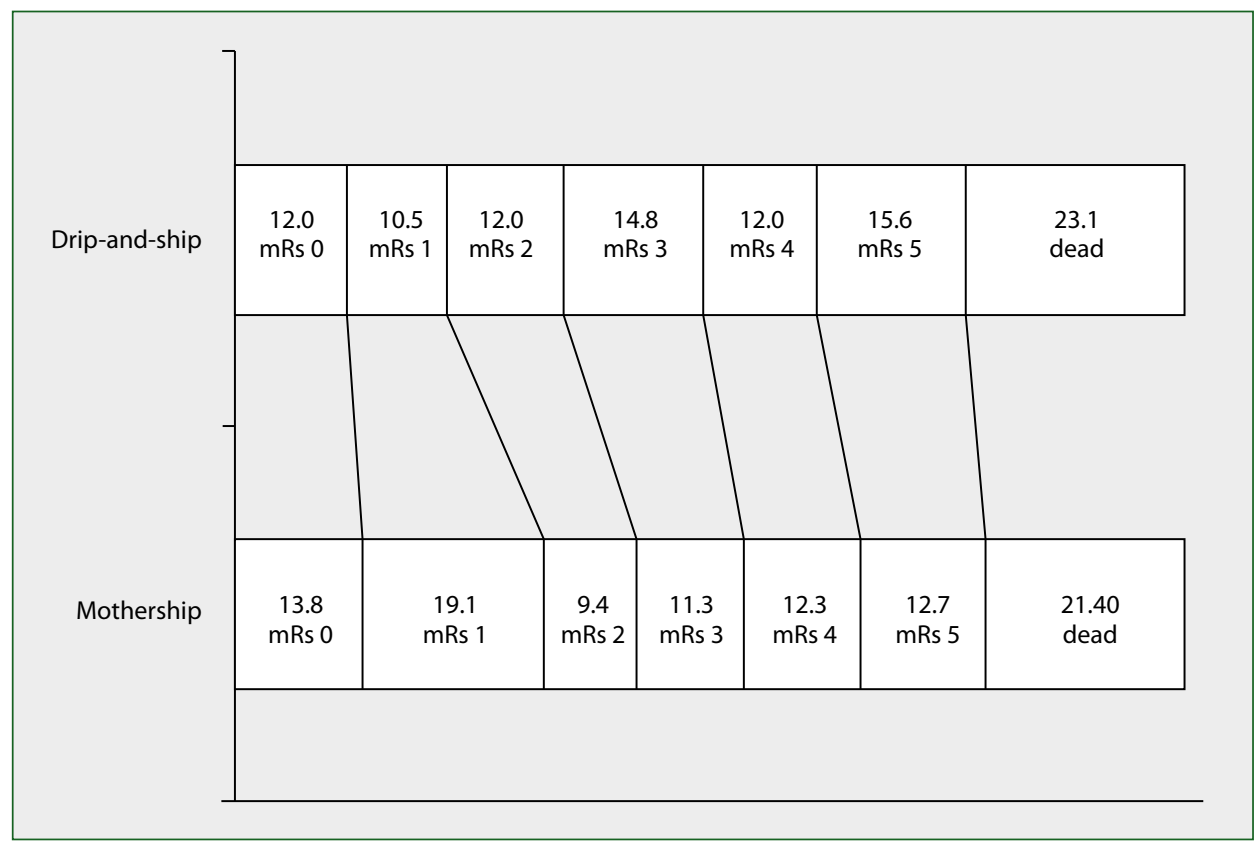

Figure 1. Percentage distribution of patients' modified Rankin scale scores at 3-month follow-up in compared groups 
DAS, $p=0.136$, Fig. 1). The rate of symptomatic intracranial haemorrhage and mortality was similar in both groups $(8.5 \%$ in MS vs. $7.9 \%$ in DAS group, $\mathrm{p}=0.101$ and $21.4 \%$ in MS vs. $23.1 \%$ in DAS group, $\mathrm{p}=0.44$, respectively).

\section{Discussion}

The aim of this study was to evaluate whether the 'mothership' paradigm featuring direct admission of a stroke patient to the CSC without prior administration of IV thrombolysis in PSC results in a higher rate of favourable clinical outcomes, as it potentially shortens the time from onset to groin puncture and may result in earlier recanalisation. Several clinical trials focusing on endovascular therapy of ischaemic stroke have highlighted the importance of rapid treatment $[7,8]$. Ota et al. [9] confirmed that onset-to-puncture time has an independent effect on functional outcomes after MT, and concluded that reducing this time metric is a key factor in successful endovascular therapy. In addition to this, the benefit of bridging IVT prior to MT is currently under discussion, and some studies have reported comparable thrombectomy outcomes with and without thrombolysis $[10,11]$.

The available studies comparing the results of MT in patients treated in the MS and DAS models do not clearly indicate an advantage for either of them. According to Garchenfeld et al., who compared both paradigms, there was no statistically significant difference either in terms of successful recanalisation or in long-term clinical outcome [14]. Similarly, the results of the RACECAT trial did not support the hypothesis that direct transfer of LVO suspected patients to CSC leads to improved outcomes [2].

On the other hand, Rinaldo et al., who reported clinical outcomes of 8,500 patients with LVO, showed that patients transferred from PSC have an increased risk of mortality compared to patients treated in CSC [12]. Recently published insights from the Ischaemic Stroke Registry in France showed that significantly more functional independence was achieved among MS patients compared to DAS patients [13].

This aligns with our findings. We observed a significant difference in 3-month excellent clinical outcomes expressed as mRS 0-1 between MS patients (32.9\%) and DAS patients (22.5\%). We also noticed that a greater number of patients achieved mRS 0-2 in the MS model (42.3\%) compared to the DAS model (34.5\%), although statistical significance was not reached. This result is most likely due to the significant onset-to-puncture time difference between the two groups (-85 min in MS patients vs. DAS patients), and was not abolished by significantly longer door-to-puncture time in the MS model compared to the DAS model.

We can assume that further acceleration of acute stroke patient management in CSCs and a 24-hour on-site angio suite team would significantly reduce the door-to-puncture time, resulting in even better treatment outcomes in the MS model. However, we should be aware that one limitation of the
MS model is the possibility of overloading CSCs with stroke patients who will not be candidates for MT.

Our study has several limitations: a) the limited number of evaluated patients which limits the generalisability of our findings; b) the retrospective design of the study; c) the data collected in the tertiary hospital in the Lublin operational area and our findings may not be relevant to other regions; and d) our study does not take into account stroke-suspected patients transferred directly to CSC but who are eventually not eligible for MT.

\section{Conclusions}

Our study shows that patients with LVO transferred directly to a CSC (the MS model) achieved a better rate of excellent 3-month functional independence expressed as $\mathrm{mRS}$ 0-1 compared to the DAS paradigm. However, if the favourable clinical outcome is defined as mRS $0-2$, the benefit of the MS approach was still not clearly demonstrated.

\section{Clinical implications}

The question of the overall best treatment paradigm for stroke patients remains unanswered. An effective and reliable pre-hospital assessment for LVO may condition the direct transport of the patient to the CSC, resulting in better clinical outcomes after MT. The use of the MS model or the DAS model should be flexible and based on the regional stroke service.

Conflict of interest: None.

Funding: None.

\section{References}

1. Ismail M, Armoiry X, Tau N, et al. Mothership versus drip and ship for thrombectomy in patients who had an acute stroke: a systematic review and meta-analysis. J Neurointerv Surg. 2019; 11(1): 14-19, doi: 10.1136/neurintsurg-2018-014249, indexed in Pubmed: 30297541.

2. Ribo M (2020) RACECAT results presented at ESO-WSO 2020. https:// eso-wso-conference.org/wp-content/uploads/sites/42/2020/11/ News-from-ESO-WSO-2020-Conference-PR1_Eng.pdf (April 3 2021).

3. Romoli M, Paciaroni M, Tsivgoulis G, et al. Mothership versus drip-andship model for mechanical thrombectomy in acute stroke: A systematic review and meta-analysis for clinical and radiological outcomes. J Stroke. 2020; 22(3): 317-323, doi: 10.5853/jos.2020.01767, indexed in Pubmed: 33053947.

4. Schlemm L, Endres M, Nolte CH. Bypassing the closest stroke center for thrombectomy candidates: what additional delay to thrombolysis is acceptable? Stroke. 2020; 51(3): 867-875, doi: 10.1161/STROKEAHA.119.027512, indexed in Pubmed: 31964288.

5. Błażejewska-Hyżorek B, Czernuszenko A, Członkowska A, et al. Ischemic stroke guidelines. Polski Przegląd Neurologiczny. 2019; 15: 53-55.

6. Larrue V, von Kummer R R, Müller A, et al. Risk factors for severe hemorrhagic transformation in ischemic stroke patients treated with recombinant tissue plasminogen activator: a secondary analysis of the European-Australasian Acute Stroke Study (ECASS II). Stroke. 
2001; 32(2): 438-441, doi: 10.1161/01.str.32.2.438, indexed in Pubmed: 11157179.

7. Khatri P, Yeatts SD, Mazighi M, et al. IMS III Trialists. Time to angiographic reperfusion and clinical outcome after acute ischaemic stroke: an analysis of data from the Interventional Management of Stroke (IMS III) phase 3 trial. Lancet Neurol. 2014; 13(6): 567-574, doi: 10.1016/S1474-4422(14)70066-3, indexed in Pubmed: 24784550.

8. Fransen PSS, Berkhemer OA, Lingsma HF, et al. Multicenter Randomized Clinical Trial of Endovascular Treatment of Acute Ischemic Stroke in the Netherlands Investigators. Time to reperfusion and treatment effect for acute ischemic stroke: A randomized clinical trial. JAMA Neurol. 2016; 73(2): 190-196, doi: 10.1001/jamaneurol.2015.3886, indexed in Pubmed: 26716735.

9. Ota T, Nishiyama Y, Koizumi S, et al. Impact of onset-to-groin puncture time within three hours on functional outcomes in mechanical thrombectomy for acute large-vessel occlusion. Interv Neuroradiol. 2018; 24(2): 162-167, doi: 10.1177/1591019917747247, indexed in Pubmed: 29237321.

10. Coutinho JM, Liebeskind DS, Slater LA, et al. Combined intravenous thrombolysis and thrombectomy vs thrombectomy alone for acute ischemic stroke: A pooled analysis of the SWIFT and STAR stud- ies. JAMA Neurol. 2017; 74(3): 268-274, doi: 10.1001/jamaneurol.2016.5374, indexed in Pubmed: 28097310.

11. Fischer U, Kaesmacher J, Mendes Pereira V, et al. Direct mechanical thrombectomy versus combined intravenous and mechanical thrombectomy in large-artery anterior circulation stroke: A topical review. Stroke. 2017; 48(10): 2912-2918, doi: 10.1161/STROKEAHA.117.017208, indexed in Pubmed: 28887391.

12. Rinaldo L, Brinjikji W, McCutcheon BA, et al. Hospital transfer associated with increased mortality after endovascular revascularization for acute ischemic stroke. J Neurointerv Surg. 2017; 9(12): 1166-1172, doi: 10.1136/neurintsurg-2016-012824, indexed in Pubmed: 27986846.

13. Weisenburger-Lile D, Blanc R, Kyheng M, et al. on behalf of the Endovascular Treatment in Ischemic Stroke Investigators. Direct admission versus secondary transfer for acute stroke patients treated with intravenous thrombolysis and thrombectomy: insights from the endovascular treatment in ischemic stroke registry. Cerebrovasc Dis. 2019; 47(3-4): 112-120, doi: 10.1159/000499112, indexed in Pubmed: 31063998.

14. Gerschenfeld G, Muresan IP, Blanc R, et al. Two paradigms for endovascular thrombectomy after intravenous thrombolysis for acute ischemic stroke. JAMA Neurol. 2017; 74(5): 549-556, doi: 10.1001/ jamaneurol.2016.5823, indexed in Pubmed: 28319240. 